\title{
Theoretical concepts of unlimited-power reflectors, absorbers, and emitters with conjugately matched layers
}

\author{
Constantinos A. Valagiannopoulos ${ }^{1}$ and Sergei A. Tretyakov ${ }^{2}$ \\ ${ }^{1}$ Department of Physics, School of Science and Technology, Nazarbayev University, KZ-010000, Astana, Kazakhstan \\ ${ }^{2}$ Department of Radio Science and Engineering, School of Electrical Engineering, Aalto University, P. O. Box 13000, FI-00076 Aalto, Finland \\ (Received 20 April 2016; revised manuscript received 8 August 2016; published xxxxxx)
}

\begin{abstract}
Recently, it was shown that by using special artificial materials it is possible to ensure that all electromagnetic modes of free space are conjugately matched to the modes of a material body and, thus, all modes deliver power to the body in the most effective way. Such a fascinating feature is acquired because the conjugate matching does not concern only the propagating modes but, most importantly, is applied to all evanescent modes; in this way, all the possible ways of transferring the electromagnetic energy to the material body can be optimally exploited. However, coupling to higher-order (mostly evanescent) modes is weak and totally disappears in the limit of an infinite planar boundary. Here, we show that by properly perturbing the surface of the receiving or emitting body with, for example, randomly distributed small particles, we can open up channels for super-radiation into the far zone. The currents induced in the small particles act as secondary sources (radiation "vessels") which send the energy to travel far away from the surface and, reciprocally, receive power from far-located sources. For a particular example, we theoretically predict about 20-fold power transfer enhancement between the conjugately matched power-receiving body (as compared with the ideal black body) and far-zone sources. Reciprocally, the proposed structure radiates about 20 times more power into the far zone as compared with the same source over a perfect reflector.
\end{abstract}

DOI: 10.1103/PhysRevB.00.005100

\section{INTRODUCTION}

The problem of optimizing and maximizing absorption and emission of electromagnetic energy is relevant for a broad variety of applications such as antennas, radar absorbers, thermal emitters and accumulators, photovoltaic devices, and more [1-8]. For macroscopic bodies (having sizes large compared to the wavelength of electromagnetic radiation), it is usually assumed that the ultimate absorber and emitter is the ideal black body that completely absorbs all incident rays (e.g., [9]). Conceptually, ideal black body, introduced by Kirchhoff [10], is totally opaque and has zero reflection coefficient for any propagating plane wave (any incidence angle and any polarization); in this sense, it is the perfect absorber of electromagnetic energy. Following the Planck theory of thermal radiation [11], the ideal black body appears to be also the ultimate thermal emitter for radiating heat into free space. Practical realization of bodies whose properties mimic those of ideal black bodies is a scientific and technical challenge (see, e.g., [2,3]).

However, it has been recently demonstrated that, in principle, it is possible to engineer bodies which can absorb power not only from incident propagating waves (incident rays in the Kirchhoff black-body concept), but also from external evanescent fields or high-order spherical harmonics of the incident-wave spectrum $[12,13]$. Due to the resonant nature of surface modes of these superabsorbing and superemitting bodies, their absorption cross section grows without limit when the medium parameters approach the ideal values, and the thermal spectral emissivity at the resonance frequency becomes arbitrarily high compared to Planck's black body of the same size and the same temperature. The material structures proposed in $[12,13]$ realize the ideas of conjugate matching of all modes of free space to all modes of the absorbing/emitting body $[8,14,15]$, and we call them conjugately matched bodies or layers (CML).
The material realizations proposed in [12,13] are based 58 on the use of double-negative (DNG) isotropic or uniaxial 59 media which obey the uniaxial perfectly matched layer (PML) 60 conditions [16]. High-order modes of conjugately matched 61 bodies $[12,13]$ resonate with all modes of free space and most 62 effectively exchange energy with them. However, to effectively 63 absorb or emit all the modes, it is necessary that the modes 64 of the body are sufficiently coupled with the corresponding 65 modes of free space. In [12], it is shown that if a conjugately 66 matched body fills a half-space (a planar infinite interface with 67 free space), its properties are the same as of the conventional 68 ideal black body and no absorption or radiation enhancement 69 over the ideal black-body limit can take place. To couple with 70 higher-order free-space modes, we can make the surface not 71 planar, and in [12] it is shown that for bodies of finite sizes, 72 on an example of a sphere, unlimited power exchange power 73 is indeed possible. An alternative scenario was explored in 74 paper [13], where the conjugately matched body filled a half- 75 space with an infinite planar interface, but the sources in free 76 space were positioned close to the interface and created large 77 evanescent fields which directly couple to the resonant surface 78 modes of the conjugately matched layer. Also in this case it 79 was seen that the absorption in the body was dramatically 80 stronger than in an ideal black body at the same position. $\quad{ }_{81}$

In this paper, we show that it is possible to realize an infinite 82 and planar surface which can absorb and emit more power than 83 the ideal black body by perturbing the surface of a conjugately 84 matched layer, introduced in [13]. In this scenario, small 85 subwavelength scatterers randomly distributed over the body 86 surface offer necessary coupling between high-order resonant ${ }_{87}$ surface modes and the far-zone fields, opening channels for 88 extra absorption or emission of energy. In the limit of ideal 89 material parameters, this planar interface not only absorbs 90 or reflects all incident propagating waves, but does the same 91 also for all evanescent harmonics. We show that a perturbed 92 
93 interface with a low-loss conjugately matched body acts as a ${ }_{94}$ "super-reflector" of fields developed from a small antenna in 95 its vicinity by launching the energy stored in the antenna near 6 field into space.

In particular, we introduce a random grid of electrically thin cylinders close to a resonant interface with a conjugately 99 matched layer, where huge reactive energy is stored. Inevitably,

currents induced in thin conductive cylinders radiate into far zone as linear antennas, and we say that these cylinders act as radiation "vessels." A random and sparse enough distribution of cylinders ensures that diffuse radiation survives in the far zone and is not coherently combined into a plane wave. We test the effects of this cluster of particles on the radiation from various conjugately matched layers and conclude that for a realizable passive structure, one can achieve a stable 20-30-fold enhancement of the far-field power.

109 Such super-reflectors are extremely strongly coupled to 10 evanescent fields of external sources and can extract power 1 from them in the most efficient way. In the antenna ter2 minology, the effective area of the CML reflector is larger 3 than the geometrical one, although the reflector size is very 14 large compared with the wavelength. Basically, we aim at 15 realization of a surface which (at its resonant frequency) would be "more reflective than the ideal perfectly conducting 17 mirror," and this property would hold even in the limit of the 18 infinite planar reflector. If the perturbing elements are lossy, 19 instead of enhancing reflection we can enhance absorption in 120 a planar absorbing layer or enhance thermal radiation from 121 a planar hot surface into the far zone beyond the Planck 22 limit of the ideal black body. We expect that perturbing the 23 surface can be a more effective mean to couple to far-zone 24 field as compared to curved surfaces. In the study [12] it was 25 expectedly found that for large spherical bodies, when the 126 curvature of the surface becomes small, one needs extreme 27 (low-loss) values of material parameters in order to realize 28 enough effective coupling to high-order harmonics. The sur29 face perturbation approach, introduced here, does not have this 30 limitation.

\section{CONJUGATELY MATCHED LAYER (CML)}

We begin the study by a brief explanation of the concept of 133 the conjugately matched layer, introduced in [13]. It has been 134 recently reported $[12,13]$ that there can exist material bodies 135 which optimally absorb energy of electromagnetic fields, by 136 achieving conjugate matching for every free-space mode. In 137 the theoretical limit of negligible losses in the absorbing 138 body, such an optimally designed finite-sized body can absorb 139 the whole infinite energy of an incident propagating plane 140 wave [12]. In [13], a uniaxial medium with special values 141 for its constituent parameters has been suggested as possible 142 realization. The permittivities and permeabilities (transversal 143 with subscript $t$ and normal to the material sample boundary 144 with subscript $n$ ) satisfy the uniaxial perfectly matched 145 layer (PML) conditions [16-20] and simultaneously possess 146 negative real parts as in a double-negative (DNG) medium [21], 147 contrary to the conventional uniaxial PML choice. For planar 148 interfaces and TM polarization (sole magnetic component 149 parallel to the half-space boundary), the material parameters satisfy

$$
\varepsilon_{t}=\mu_{t}=\frac{1}{\varepsilon_{n}}=a-j b,
$$

where $a$ and $b$ are real parameters and $a<0$. We assume 151 harmonic time dependence $\exp (+j \omega t)$, where $\omega$ is the angular 152 operating frequency and $j$ is the imaginary unit. The parameter 153 $b>0$ corresponds to losses for propagating plane waves, 154 and it is easy to show [16,26] that sufficiently thick slabs 155 of such materials behave as perfect absorbers for arbitrary 156 incident propagating plane waves. From duality, a similar 157 expression for the parameters of uniaxial perfect absorbers 158 holds for the fields of the TE polarization: $\varepsilon_{t}=\mu_{t}=\frac{1}{\mu_{n}}={ }_{159}$ $a-j b$. To ensure that the thought properties hold for both 160 orthogonal polarizations, we can require that $\varepsilon_{n}=\mu_{n}$. For 161 compactness, in the following we present only formulas for ${ }_{162}$ the TM polarization, without compromising the generality. 163

Any uniaxial medium characterized by the constituent 164 parameters $\left(\varepsilon_{t}, \mu_{t}, \varepsilon_{n}\right)$ has the following TM wave impedance 165 $Z$ (e.g., [26]):

$$
Z=-j \frac{\eta_{0}}{k_{0} \varepsilon_{t}} \sqrt{\frac{\varepsilon_{t}}{\varepsilon_{n}} k_{t}^{2}-\varepsilon_{t} \mu_{t} k_{0}^{2}},
$$

which relates the tangential to the interface components of ${ }_{167}$ electric and magnetic fields of plane waves in the medium. 168 The notations $\eta_{0}=\sqrt{\mu_{0} / \varepsilon_{0}}$ and $k_{0}=\omega \sqrt{\varepsilon_{0} \mu_{0}}=2 \pi / \lambda_{0}$ cor- ${ }_{169}$ respond to the free-space wave impedance and wave number, 170 respectively ( $\varepsilon_{0}$ and $\mu_{0}$ are the permittivity and permeability of 171 vacuum, while $\lambda_{0}$ is the operational wavelength in free space). ${ }_{172}$ The symbol $k_{t}$ is used for the transversal wave number of the 173 incident plane wave. Vector $\mathbf{k}_{t}$ is parallel to the boundary of 174 the half-space and normal to the sole component of magnetic 175 field. The basic property of a material with the constituent 176 parameters given by (1) when $a<0$ is that its wave impedance ${ }_{177}$ $Z$ is the complex conjugate of the TM wave impedance of 178 vacuum $Z^{*}=Z_{0}=-j \frac{\eta_{0}}{k_{0}} \sqrt{k_{t}^{2}-k_{0}^{2}}$ [13].

Most importantly, this equality is valid for every real wave 180 number $k_{t} \in \mathbb{R}$, either of a propagating wave in free space 181 $\left(\left|k_{t}\right|<k_{0}\right)$ or of an evanescent mode $\left(\left|k_{t}\right|>k_{0}\right)$. Therefore, 182 the use of such conjugately matched layers (CLM), as we ${ }_{183}$ call them, leads to maximal power transfer from arbitrary 184 incident fields into the medium since they optimally use every 185 possible way (mode) available from sources outside of the 186 material sample. Actually, in this ideal case of overall lossless 187 conjugately matched medium, fields tend to infinity at the 188 material surface. Assuming infinitesimally small losses in the 189 CML, infinite power can be delivered to the medium, provided 190 that the incident evanescent field is created by an antenna fed 191 by an ideal voltage or current source, capable of supplying 192 infinite power. In other words, the CML structure is identical 193 to ordinary PML for $\left|k_{t}\right|<k_{0}$ but operates totally differently 194 for $\left|k_{t}\right|>k_{0}$ aiming not at zero reflection but at the maximal 195 power transfer.

By inspection of (1), one can directly infer that if $b>0,{ }_{197}$ the transversal relative constituent parameters $\varepsilon_{t}, \mu_{t}$, are lossy; 198 however, the normal component of the permittivity $\varepsilon_{n}$ is an 199 active one. In order to identify the overall character of the 200 uniaxial medium, we consider a perturbed version of the 201 ideal material parameter values by using a small additional 202 
(a)

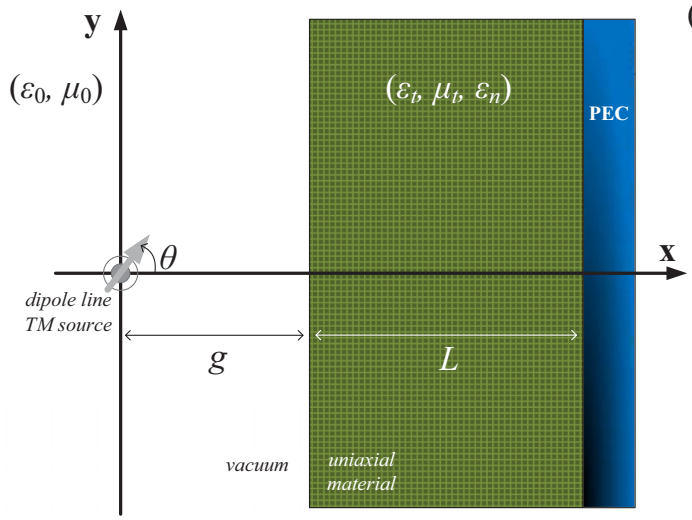

(b)

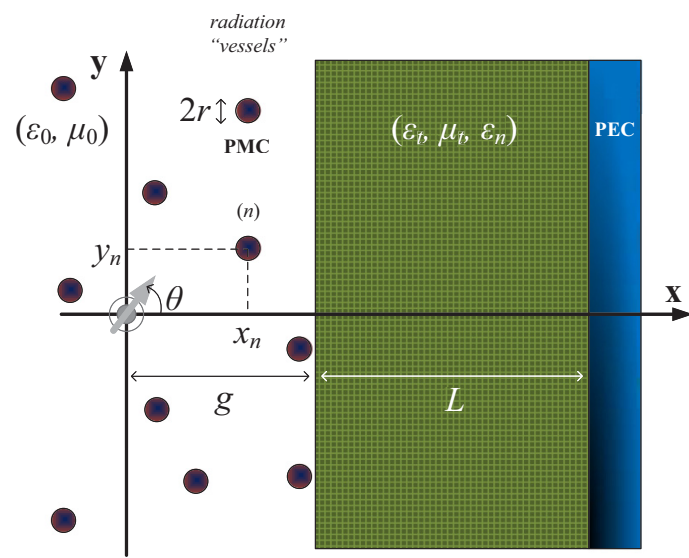

FIG. 1. (a) The testbed configuration of a grounded electrically thick slab filled with a uniaxial material $\left(\varepsilon_{t}, \mu_{t}, \varepsilon_{n}\right)$ with the thickness $L$, excited by a TM electric dipole line inclined by the angle $\theta$ located at a small distance $g$ from the air-medium interface. (b) The same structure in the presence of a cluster of $N$ electrically thin (the radius $r$ ) circular perfect magnetic conductor (PMC) cylinders randomly distributed in the vicinity of the air-slab interface with arbitrary coordinates $\left(x_{n}, y_{n}\right)$ for $n=1, \ldots, N$.

203 parameter $\delta$ controlling the imaginary part of the normal 204 permittivity $\varepsilon_{n}=\frac{1}{\varepsilon_{t}}-j \delta=\frac{1}{a-j b}-j \delta$, which tends to the 205 ideal CML medium parameters for $\delta \rightarrow 0$. To study the 206 properties of such a quasi-CML medium, we use the testbed 207 configuration illustrated in Fig. 1(a). For simplicity of analyt208 ical considerations, we assume that there is no dependence on 209 one of the tangential coordinates $(z)$. A grounded slab of the 210 thickness $L$, filled with a uniaxial material with the constituent 211 parameters $\left(\varepsilon_{t}, \mu_{t}, \varepsilon_{n}\right)$, is excited by an infinite electric-dipole 212 line located at the distance $g$ from the air-medium interface. 213 The exciting dipole moments are orthogonal to the axis $z$ 214 and inclined by the angle $\theta$ with respect to the axis $x$ 215 [Fig. 1(a)]. In this configuration, the magnetic field has only 216 one nonzero component (along $z$ ) and the fields are TM 217 polarized.

218 In [13], an approximate analytical formula for the absorbed 219 power (per unit length along the $\hat{\mathbf{z}}$ axis) has been derived. It 220 shows that the absorbed power $P$ is a sum of two terms. The first 221 term corresponds to the power delivered by the propagating 222 modes $P_{\text {prop }}=\mu_{0} \omega^{3} q^{2} / 16$, and it is independent from the 223 angle $\theta$. Here, $q$ is the electric dipole moment per unit length 224 of the line (measured in Coulomb). The second term gives 225 the power absorbed from the evanescent-modes fields and is 226 written as [13]

$$
\begin{aligned}
P_{\text {evan }} \cong & P_{\text {prop }} \frac{8|a|}{k_{0}^{2} \pi} \int_{k_{0}}^{+\infty} \frac{k_{t}^{2}\left(k_{t}^{2}-k_{0}^{2} \sin ^{2} \theta\right)}{\left(k_{t}^{2}-k_{0}^{2}\right)^{3 / 2}} e^{-2 g \sqrt{k_{t}^{2}-k_{0}^{2}}} \\
& \times \frac{\delta}{[1+\operatorname{sgn}(a)]^{2}+\delta^{2}\left[\frac{k_{t}^{2}\left|\varepsilon_{t}\right|}{2\left(k_{t}^{2}-k_{0}^{2}\right)}\right]^{2}} d k_{t}
\end{aligned}
$$

227 for $\delta \rightarrow 0$. It is noteworthy that the CML slab acts as an 228 ultraefficient passive absorber $(P \rightarrow+\infty)$ of the incoming 229 illumination for $\delta>0$ and as an infinite-power active emitter 230 $(P \rightarrow-\infty)$ for $\delta<0$. In the limit of $\delta \rightarrow 0^{+}$, both the 231 field strength at the surface and the absorbed power diverge 232 and tend to infinity. Therefore, it would be meaningful to 233 inspect the field distributions leading to such unbounded field 234 concentrations.

\section{EXCITATION OF CML}

Let us examine the fields created by a small source in the ${ }_{236}$ vicinity of an infinite and homogeneous CML slab within the 237 testbed setup shown in Fig. 1(a). The corresponding boundary- 238 value problem is scalar, and the magnetic field possesses a 239 sole component parallel to $\hat{\mathbf{z}}$ axis $[\mathbf{H}=\hat{\mathbf{z}} H(x, y)]$. The used 240 Cartesian coordinate system $(x, y, z)$ is also defined in Fig. 1(a), 241 with the primary dipole line source positioned at $(x, y)=(0,0)$. 242 The incident magnetic field from that electric-dipole line 243 (existing in vacuum) can be expressed in the following integral 244 form [22]:

$$
\begin{aligned}
H_{\text {inc }}(x, y)= & -\frac{\omega q}{4 \pi} \int_{-\infty}^{+\infty} e^{-|x| \kappa_{0}\left(k_{t}\right)} \\
& \times\left[\frac{k_{t}}{\kappa_{0}\left(k_{t}\right)} \cos \theta+j \sin \theta \operatorname{sgn}(x)\right] e^{-j k_{t} y} d k_{t},
\end{aligned}
$$

where the normal to the interface component of the wave 246 number $\kappa_{0}\left(k_{t}\right)=\sqrt{k_{t}^{2}-k_{0}^{2}}$ is evaluated with a positive real 247 part and if the real part is zero, as a positive imaginary number. 248 Analytical expression for the incident field involving Hankel 249 function [22,23] is also available but not given here for brevity 250 since all the field quantities are expressed as spectral integrals. 251

The formulated boundary-value problem can be solved 252 analytically. As a result, we find that the secondary field 253 developed due to the presence of the uniaxial slab and the 254 PEC plane in free space $(x<g)$ is given by $H_{\mathrm{sec}}(x, y)=255$ $\int_{-\infty}^{+\infty} S_{\mathrm{sec}}\left(k_{t}\right) e^{\kappa_{0}\left(k_{t}\right) x-j k_{t} y} d k_{t}$, where 256

$$
\begin{aligned}
S_{\mathrm{sec}}\left(k_{t}\right)= & \frac{\omega q}{4 \pi} e^{-2 g \kappa_{0}\left(k_{t}\right)}\left(\frac{k_{t}}{\kappa_{0}\left(k_{t}\right)} \cos \theta+j \sin \theta\right) \\
& \times \frac{\kappa\left(k_{t}\right)-\varepsilon_{t} \operatorname{coth}\left[\kappa\left(k_{t}\right) L\right] \kappa_{0}\left(k_{t}\right)}{\kappa\left(k_{t}\right)+\varepsilon_{t} \operatorname{coth}\left[\kappa\left(k_{t}\right) L\right] \kappa_{0}\left(k_{t}\right)} .
\end{aligned}
$$

The value of

$$
\kappa\left(k_{t}\right)=\sqrt{k_{t}^{2} \frac{\varepsilon_{t}}{\varepsilon_{n}}-k_{0}^{2} \varepsilon_{t} \mu_{t}}
$$



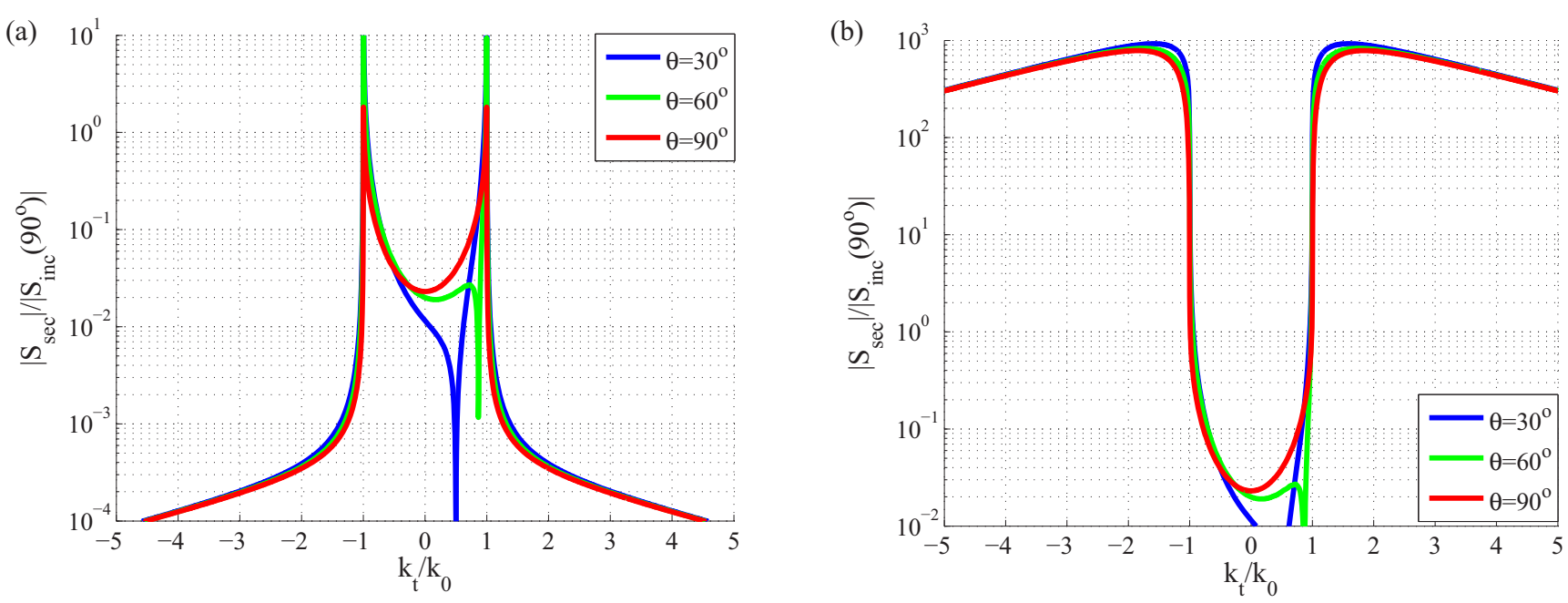

FIG. 2. The magnitude of the spatial spectral density function of the secondary field $S_{\mathrm{sec}}\left(k_{t}\right)$ at $x=0$ with respect to the normalized wave number $k_{t} / k_{0}$ for various inclination angles $\theta$ and (a) $a=2$ (DPS-PML) and (b) $a=-2$ (CML). Common plot parameters: $b=0.1, \delta=0.001$, $g=0.03 \lambda_{0}, L=3 \lambda_{0}$. The represented quantity is normalized by $S_{\text {inc }}\left(90^{\circ}\right)=j \omega q /(4 \pi)$.

258 is the normal component of the plane-wave propagation 259 constant in the CML slab. The total field in vacuum equals 260 to $H_{\text {back }}(x, y)=H_{\text {inc }}(x, y)+H_{\text {sec }}(x, y)$.

261 In Fig. 2, we present the magnitude of the integrand in 262 the formula of the secondary magnetic field for $x=0$ (very 263 close to the air-CML boundary located at $x=g$ ), equal to $264\left|S_{\mathrm{sec}}\left(k_{t}\right)\right|$ as a function of the normalized transversal wave 265 number $k_{t} / k_{0}$ for various inclination angles of the source $266 \theta$. The presented quantity is normalized by the (constant) 267 magnitude of the integrand of the incident field (4) for $\theta=90^{\circ}$ : ${ }_{268} S_{\text {inc }}\left(90^{\circ}\right)=\frac{j \omega q}{4 \pi}$, which is independent from $k_{t}$ and gives us a 269 metric of the incident power. Figure 2(a) corresponds to a 270 double-positive (DPS) conventional uniaxial PML [16] and 271 it is directly observed that $\left|S_{\mathrm{sec}}\right|$ vanishes exponentially for 272 evanescent waves $\left(\left|k_{t}\right|>k_{0}\right)$. On the contrary, for the CML case [Fig. 2(b)], the integrand values have huge magnitudes 273 for $\left|k_{t}\right|>k_{0}$ regardless of the angle $\theta$. These graphs verify 274 the aforementioned theoretical expectation that unbounded 275 absorbed power of (3) in the CML case $(a<0$ and $|\delta| \rightarrow 0)$ is 276 due to the extremely large magnitudes of the evanescent fields 277 developed in the vicinity of the interface, as demonstrated by 278 Fig. 2(b). Note the different scale in Figs. 2(a) and 2(b): the 279 values in the region $-1<k_{t} / k_{0}<1$, which correspond to the 280 propagating-wave part of the spectrum, are the same in both 281 figures.

Figure 3 shows the spatial distribution of the total magnetic 283 field $\left|H_{\text {back }}(x, y)\right|$ for the two cases of Fig. 2 (with $\theta=90^{\circ}$ ). ${ }_{284}$ The represented quantity is normalized by the value of 285 the incident field at $(x, y)=(g, 0)$ and is expressed in $d B .286$ We again observe how more efficient is the CML medium 287 (a)

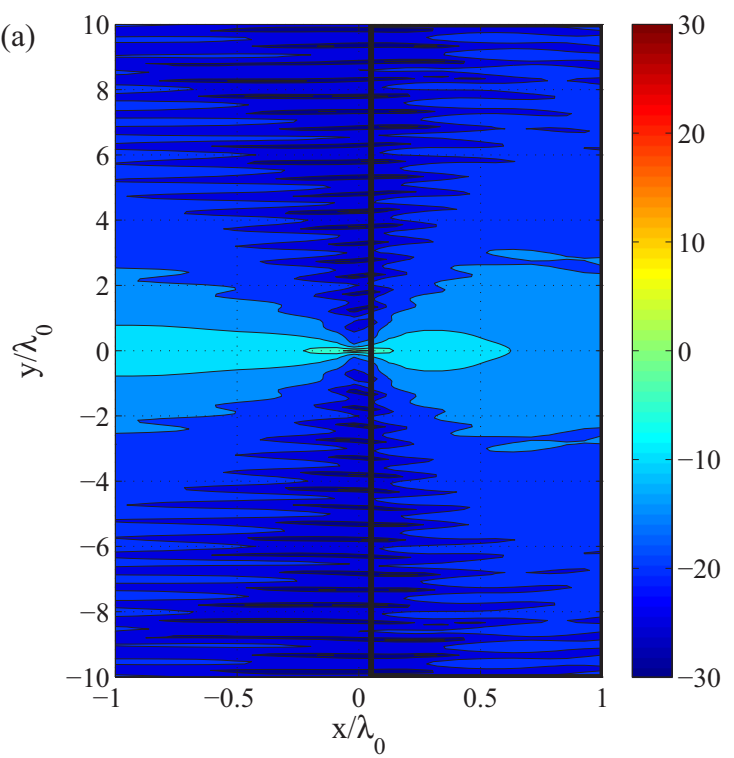

(b)

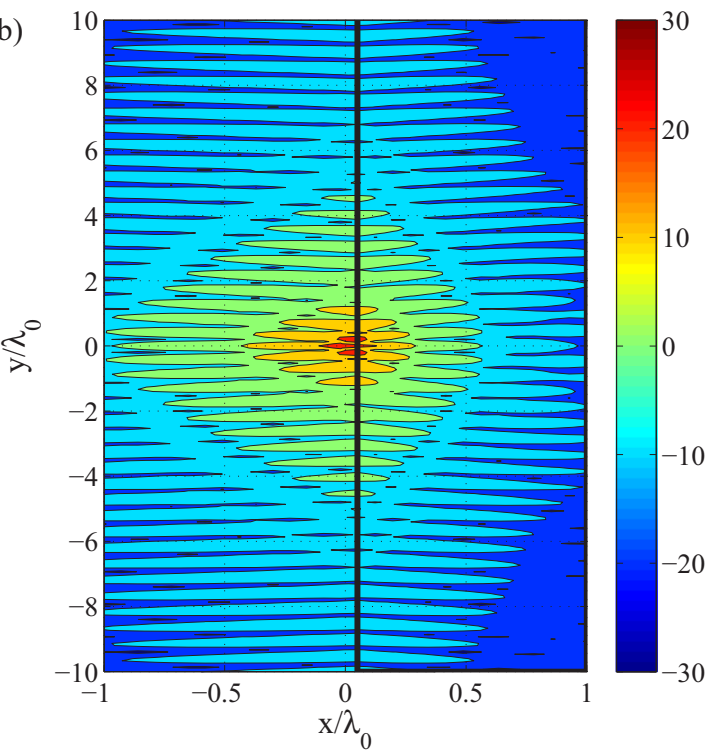

FIG. 3. The spatial distributions of the total magnetic field $H_{\text {back }}(x, y)$ normalized by $H_{\text {inc }}(g, 0)$ expressed in $d B$ for (a) $a=2$ (DPS-PML) and (b) $a=-2$ (CML). Common plot parameters: $b=0.1, \delta=0.001, g=0.03 \lambda_{0}, L=3 \lambda_{0}, \theta=90^{\circ}$. 
288 [Fig. 2(b)] in exciting fields along its boundary compared to 289 the conventional PML case [Fig. 2(a)]. However, since the 290 nature of these fields is evanescent, they are rapidly decaying 291 with increasing the distance from the surface $(x \rightarrow-\infty)$. It 292 should be stressed that the concentration of the fields in the 293 vicinity of the CML interface is always huge regardless of the 294 sign of $\delta$, both for overall passive $(\delta>0)$ or active $(\delta<0)$ 295 structures.

In this paper, we propose to make use of this concentration 297 of fields along the boundary of the two regions $(x=g)$ to 298 create an "antenna," which would "launch" the energy stored 299 in this region into the far zone $x \rightarrow-\infty$. This is not an easy 300 task, though. It is well known that resonant surface modes 301 along infinite and regular surfaces do not radiate energy into 302 the far zone. In other words, despite the huge difference of 303 the two systems (PML versus CML slab) in the near field, 304 the behavior of the field radiated in the far region is similar. 305 Actually, with the use of the stationary phase method, one can 306 directly evaluate the azimuthal profiles of the incident and the 307 secondary fields in the far zone as follows:

$$
\begin{gathered}
h_{\text {inc }}(\varphi) \sim \frac{k_{0} j \omega q}{4} \sin (\varphi-\theta), \quad x \rightarrow-\infty, \\
h_{\mathrm{sec}}(\varphi) \sim-\pi k_{0} S_{\mathrm{sec}}\left(k_{0} \sin \varphi\right) \cos \varphi, \quad x \rightarrow-\infty,
\end{gathered}
$$

308 for $90^{\circ}<\varphi<270^{\circ}$. We notice that the expression of the 309 secondary component, which describes the effect of the 310 grounded slab, is proportional to a specific value of the function ${ }_{311} S_{\mathrm{sec}}\left(k_{t}\right)$ : the one corresponding to $k_{t}=k_{0} \sin \varphi$. Since this 312 value is always smaller in magnitude than $k_{0}(\varphi \in \mathbb{R})$, namely, 313 corresponding to a propagating and not to an evanescent mode, 314 it is clear that huge reactive fields of Fig. 2(b) do not to 315 contribute to far-zone radiation. The situation is not the same 316 if one uses as an electromagnetic energy sink a finite-size 317 body filled with a suitable CML medium. In cylindrical [13] or 318 spherical [12] cases, there are no purely evanescent modes and 319 thus all the fields contribute (poorly) to the radiative power. In 320 the infinite slab case analyzed here, the necessity of something 321 that can act as a radiation "vessel" to allow the field energy 322 stored in resonant surface modes to propagate far away from 323 the source, becomes clear.

\section{RADIATION WITH "VESSELS"}

\section{A. Circuit theory approach}

In an attempt to find a way to exploit this huge field 327 concentration and transform the sizable magnitude of evanes328 cent modes (developed close to $x=g$ ) into radiative fields, 329 we consider the configuration of Fig. 1(b). Let us randomly 330 distribute small cylindrical scatterers in the vicinity of the 331 air-CML slab interface. It is expected that the large evanescent 332 fields would excite currents along these wires, which will act 3 з3 as radiation vessels, and their own field would be expressed as 334 cylindrical modes which are always partially propagating and 335 survive in the far region.

The idea of perturbing the surface with tiny scatterers can 337 be understood from the equivalent circuit corresponding to 338 the fields of a particular evanescent plane-wave component 339 exciting the CML slab in presence of a small scatterer, shown 340 in Fig. 4. The ideal voltage source $V$ represents the primary

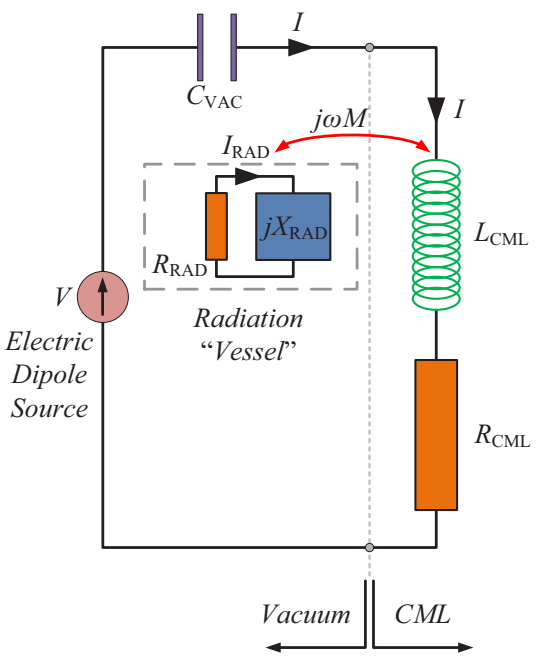

FIG. 4. Sketch of the equivalent circuit for excitation by a particular evanescent TM plane-wave component. The wave impedance of TM waves in vacuum $Z_{0}$ corresponds to the capacitance $C_{\mathrm{VAC}}$, while the impedance $Z$ of the CML contains a loss resistor $R_{\mathrm{CML}}$ and an inductive $L_{\mathrm{CML}}$ component. The cylindrical vessel in the near field of vacuum-CML interface is characterized by a radiation resistance $R_{\mathrm{RAD}}$ and a reactive impedance $X_{\mathrm{RAD}}$.

radiator (an electric dipole source in this configuration) and 341 capacitance $C_{\mathrm{VAC}}$ expresses the wave impedance of free space 342 for a specific value of $k_{t}$, given by (2) for $\varepsilon_{t}=\mu_{t}=\varepsilon_{n}=1 . \quad 343$ The inductive complex impedance $\left(R_{\mathrm{CML}}+j \omega L_{\mathrm{CML}}\right)$ is given 344 by (2) with the parameters of the CML layer. Resistance $R_{\mathrm{CML}} \quad 345$ models the dissipative losses in the CML slab and the current ${ }_{346}$ flown through the primary circuit is denoted by $I$. The small 347 scatterer (radiation "vessel") in the vicinity of the interface 348 is modeled by the radiation vessel circuit, of current $I_{\mathrm{RAD}}, \quad 349$ formed by a nonresonant reactive element $\left(j X_{\mathrm{RAD}}\right)$ (capacitive 350 or inductive) and a small resistor of the radiator $R_{\mathrm{RAD}}$. If 351 the scatterer is lossless, $R_{\mathrm{RAD}}$ corresponds to the radiation 352 resistance, and if it is absorptive, the resistance is the sum of the 353 radiative and dissipative terms. Near-field coupling between 354 the scatterer and the resonant surface mode of the interface is 355 modeled for simplicity by mutual inductance $j \omega M$. In general, 356 the mutual impedance $j \omega M$ can be a complex number with 357 any sign of the imaginary part; however, here we confine our 358 analysis to a very closely positioned particle, in which case 359 the mutual impedance is predominantly reactive $(M \in \mathbb{R})$ and 360 inductive $(M>0)$ for the considered TM polarization. $\quad 361$

The circuit in the absence of the radiation vessels $(M=0){ }_{362}$ has been analyzed in [13], and it is clear that the power 363 delivered to the loss resistor $R_{\mathrm{CML}}$ tends to infinity when the 364 series $L C$ circuit works at resonance and under the additional 365 condition $R_{\mathrm{CML}} \rightarrow 0$. In other words, the absorbed power 366 is limited only by the energy available from the primary 367 source, while there is no radiation towards the far zone 368 $\left(P_{\mathrm{RAD}}=0\right)$. In the presence of the vessels, however, the 369 systems behave dramatically different. Considering the circuit 370 of Fig. 4, we can easily find the current amplitude both in 371 the directly fed branch $(I)$ and in the circuit of the radiating 372 
$374 \operatorname{vessel}\left(I_{\text {RAD }}\right)$ :

$$
I=\frac{V\left(R_{\mathrm{RAD}}+j X_{\mathrm{RAD}}\right)}{\omega^{2} M^{2}+\left(R_{\mathrm{RAD}}+j X_{\mathrm{RAD}}\right)\left(R_{\mathrm{CML}}+j \omega L_{\mathrm{CML}}+\frac{1}{j \omega C_{\mathrm{VAC}}}\right)},
$$

$$
I_{\mathrm{RAD}}=-\frac{j \omega M V}{\omega^{2} M^{2}+\left(R_{\mathrm{RAD}}+j X_{\mathrm{RAD}}\right)\left(R_{\mathrm{CML}}+j \omega L_{\mathrm{CML}}+\frac{1}{j \omega C_{\mathrm{VAC}}}\right)} .
$$

388 under the assumption that the system works at CML resonant 389 frequency, namely, $\omega=1 / \sqrt{L_{\mathrm{CML}} C_{\mathrm{VAC}}}$. For resonant and 390 low-loss CML $\left(R_{\mathrm{CML}} \rightarrow 0\right)$, the expression for the radiated 391 power simplifies to $P_{\mathrm{RAD}}=\frac{R_{\mathrm{RAD}}|V|^{2}}{2 \omega^{2} M^{2}}$. It is clear that in order to 392 enhance radiation, we need to bring the CML to resonance 393 and reduce its losses, while the vessels can be small and 394 nonresonant. Coupling between the scatterers and the surface 395 modes should be weak in the scenario.

396 In the reciprocal situation of excitation by far-zone sources, 397 we see that it is possible to enhance absorption beyond the 398 ideal black-body full absorption of propagating plane waves 399 by making the small scatterers lossy. In this case, assuming 400 that the scatterers do not create a significant shadow for the 401 propagating modes, the propagating plane waves deliver nearly 402 all their power to the CML body, while the evanescent waves 403 (high-order cylindrical harmonics) couple to the resonant 404 surface modes via the small scatterers and deliver additional 405 power into the loss resistors of the scatterers.

\section{B. Electromagnetic theory approach}

407 Having understood the basic operational principle from an 408 equivalent circuit, which is by default an approximation for 409 every single mode $k_{t}$, we will next solve the problem rigorously 410 for the entire spectrum of $k_{t}$. A spectrum integral formulation 411 is feasible if we assume random but specific positions of a 412 finite number of scatterers $\left(x_{n}, y_{n}\right)$ on the $x y$ plane, where ${ }_{413} n=1, \ldots, N$ [as shown in Fig. 1(b)]. For the sake of simplicity 414 of test calculations, we assume that particles are circular 415 cylinders of a small radius $r$ and of perfectly magnetically 416 conducting (PMC) material. In Fig. 1(b), the pins are located 417 even at $x<0$ since we have implied that the distance $g$ of 418 the primary dipole from the interface is electrically small and 419 thus the evanescent fields may be strong even at the left side 420 of the source. Such a choice does not affect the presented

concept since similar results can be obtained if we restrict 421 the radiation vessels positions to the strip $0<x<g$. We 422 chose perfect magnetic conductor pins as a simple model of ${ }_{423}$ lossless scatterers supporting magnetic currents which is most 424 appropriate for the considered TM polarization. Conceptual 425 results will not change for any other small lossless scatterers 426 at the same positions. Green's function of the considered 427 configuration for both source $(\chi, \psi)$ and observation points 428 $(x, y)$ in vacuum is comprised of two components. The singular ${ }_{429}$ component is just a cylindrical wave [23]:

$$
G_{\text {singular }}(x, y, \chi, \psi)=-\frac{j}{4} H_{0}^{(2)}\left(k_{0} \sqrt{(x-\chi)^{2}+(y-\psi)^{2}}\right),
$$

where $H_{0}^{(2)}$ is the Hankel function of zero order and second 431 type. The smooth component of Green's function describes the 432 effect of the grounded CML slab on the free-space field and is 433 found as follows:

$$
G_{\text {smooth }}(x, y, \chi, \psi)=\int_{-\infty}^{+\infty} S_{\text {gre }}\left(k_{t}\right) e^{\kappa_{0}\left(k_{t}\right)(x+\chi)} e^{-j k_{t}(y-\psi)} d k_{t},
$$

where the spatial spectral density is given by [24]

$$
S_{\text {gre }}\left(k_{t}\right)=\frac{1}{4 \pi} \frac{e^{-2 \kappa_{0}\left(k_{t}\right) g}}{\kappa_{0}\left(k_{t}\right)} \frac{\varepsilon_{t} \operatorname{coth}\left[\kappa\left(k_{t}\right) L\right] \kappa_{0}\left(k_{t}\right)-\kappa\left(k_{t}\right)}{\varepsilon_{t} \operatorname{coth}\left[\kappa\left(k_{t}\right) L\right] \kappa_{0}\left(k_{t}\right)+\kappa\left(k_{t}\right)} .
$$

If we use the symbol $M_{n}(n=1, \ldots, N)$ for the magnetic ${ }_{436}$ currents (measured in volt/meter) induced along the axes of ${ }_{437}$ the cylinders, the scattered magnetic field produced due to the 438 presence of them is given as the following integral [25]:

$$
\begin{aligned}
H_{\text {scat }}(x, y)= & -\frac{j k_{0}}{\eta_{0}} \sum_{n=1}^{N} \int_{\left(C_{n}\right)} M_{n}(l)\left[G_{\text {singular }}(x, y, \chi(l), \psi(l))\right. \\
& \left.+G_{\text {smooth }}(x, y, \chi(l), \psi(l))\right] d l .
\end{aligned}
$$

The notation $C_{n}$ is used for the contours of cylinder's surfaces. 440 Since the cylinder radius is electrically small $\left(k_{0} r \ll 1\right)$, the ${ }_{441}$ magnetic currents can be assumed to be uniformly distributed 442 over the cylinder perimeter, and modeled by line currents 443 along the cylinder axes, namely, $M_{n}(l) \cong M_{n}$. In this way, the ${ }_{444}$ approximate boundary condition for zero total magnetic field at ${ }_{445}$ the centers of the cylinders $H_{\text {back }}\left(x_{m}, y_{m}\right)+H_{\text {scat }}\left(x_{m}, y_{m}\right)=0 \quad{ }_{446}$ for $m=1, \ldots, N$ can be enforced to formulate the following ${ }_{447}$ $N \times N$ linear system of equations with respect to the unknown 448 magnetic currents $M_{n}$ :

$$
\begin{aligned}
& \sum_{n=1}^{N} M_{n}\left[I_{m n}+2 \pi r G_{\text {smooth }}\left(x_{m}, y_{m}, x_{n}, y_{n}\right)\right] \\
& \quad=\frac{\eta_{0}}{j k_{0}} H_{\text {back }}\left(x_{m}, y_{m}\right) .
\end{aligned}
$$


450 The quantity $I_{m n}$ is the following approximately evaluated 451 integral:

$$
\begin{aligned}
I_{m n} & =\int_{\left(C_{n}\right)} G_{\text {singular }}\left(x_{m}, y_{m}, \chi(l), \psi(l)\right) d l \\
& =-\frac{j \pi r}{2}\left\{\begin{array}{l}
H_{0}^{(2)}\left(k_{0} r\right), m=n \\
H_{0}^{(2)}\left(k_{0} d_{m n}\right), m \neq n
\end{array}\right.
\end{aligned}
$$

452 where $d_{m n}=\sqrt{\left(x_{m}-x_{n}\right)^{2}+\left(y_{m}-y_{n}\right)^{2}}$ is the distance be453 tween the centers of the $n$th and the $m$ th particles.

$454 \quad$ In this way, the induced magnetic currents can be found and 455 the scattered field in the far region ( $\varphi$-dependent profile) takes 456 the form

$$
\begin{aligned}
h_{\text {scat }}(\varphi) \sim & -\frac{j 2 \pi k_{0} r}{\eta_{0}} \sum_{n=1}^{N} M_{n}\left[e^{j k_{0} \rho_{n} \cos \left(\varphi-\varphi_{n}\right)}\right. \\
& \left.-\pi k_{0} \cos \varphi S_{\text {gre }}\left(k_{0} \sin \varphi\right) e^{-j k_{0} \rho_{n} \cos \left(\varphi+\varphi_{n}\right)}\right] \\
& x \rightarrow-\infty
\end{aligned}
$$

457 where $\rho_{n}=\sqrt{x_{n}^{2}+y_{n}^{2}}$ and $\varphi_{n}=\arctan \left(x_{n}, y_{n}\right)$ are the polar 458 coordinates of the cylindrical radiation vessels. Thus, we have 459 obtained the analytical solution for the far field of the CML slab 460 in the presence of numerous radiation vessels. In the following, 461 we are going to use both approaches (circuit analysis and 462 electromagnetic analysis) in order to study, interpret, and 463 quantify the radiation enhancement achieved when the pins 464 are located in the vicinity of the vacuum-CML interface.

\section{NUMERICAL RESULTS}

466 In the following examples, we use a large number of vessels 467 which are positioned neither very close to each other, to avoid 468 building effective PMC walls which will block the incident 469 illumination, nor too far since we want a strong background 470 field at their positions. In particular, we locate $N=80$ random 471 points $\left(x_{n}, y_{n}\right)$ for $n=1, \ldots, N$ belonging to a narrow vertical 472 strip $\left\{-\lambda_{0} / 20<x<\lambda_{0} / 20,-10 \lambda_{0}<y<10 \lambda_{0}\right\}$. The dis473 tance between every couple of centers of the cylinders $d_{m n}$ is 474 kept larger than $\lambda_{0} / 5$, so that the lattice is inhomogeneous at 475 the wavelength scale and there is strong diffuse scattering into 476 the far zone [26]. As referred above, we confine ourselves to 477 uniaxial media (under TM illumination) with

$$
\varepsilon_{t}=\mu_{t}=a-j b, \varepsilon_{n}=\frac{1}{\varepsilon_{t}}-j \delta=\frac{1}{a-j b}-j \delta,
$$

478 and we are mainly interested in the CML cases with $a<0$. 479 Obviously, the presented results are dependent on the random 480 distribution of the radiation vessels; however, our studies of a 481 number of particular realizations of the pins distribution show 482 that the obtained conclusions are valid regardless of the spatial 483 distribution of the PMC pins in the vicinity of the CML slab.

\section{A. Radiation enhancement}

485 A metric of how strong is the effect of the radiation 486 vessels on the radiated far-field strength should be definitely 487 related with the energy of the azimuthal field profiles: $488\left\{h_{\text {inc }}(\varphi), h_{\text {sec }}(\varphi), h_{\text {scat }}(\varphi)\right\}$. In particular, we can define the 489 radiation enhancement ratio $R$ as the ratio of the far-zone power radiated in the presence of the near-field scatterers over 490 the corresponding quantity in the absence of them:

$$
\begin{aligned}
R & =\frac{\int_{\pi / 2}^{3 \pi / 2}|h(\varphi)|^{2} d \varphi}{\int_{\pi / 2}^{3 \pi / 2}\left|h_{\mathrm{inc}}(\varphi)+h_{\mathrm{sec}}(\varphi)\right|^{2} d \varphi} \\
& \equiv \frac{\int_{\pi / 2}^{3 \pi / 2}\left|h_{\mathrm{inc}}(\varphi)+h_{\mathrm{sec}}(\varphi)+h_{\mathrm{scat}}(\varphi)\right|^{2} d \varphi}{\int_{\pi / 2}^{3 \pi / 2}\left|h_{\mathrm{inc}}(\varphi)+h_{\mathrm{sec}}(\varphi)\right|^{2} d \varphi} .
\end{aligned}
$$

Here, we evaluate and analyze the radiation enhancement fac- 492 tor $R$ when certain parameters of the considered configuration 493 vary. We are seeking for combinations of the structure, the 494 material parameters, and the excitation which lead to $R \gg 1,495$ namely, a substantial improvement of the radiated power when 496 one puts a random cluster of cylinders in the near region of the 497 resonant surface.

In Fig. 5(a), we show the ratio $R$ as a function of the real part ${ }_{499}$ $a$ of the relative transversal permittivities/permeabilities $(a=500$ $\left.\operatorname{Re}\left[\varepsilon_{t}\right]=\operatorname{Re}\left[\mu_{t}\right]\right)$ for various perturbation parameters $\delta$. One 501 directly observes a huge change in the magnitude of $R$ taking 502 place when the material parameters transit from the double- 503 negative $(\mathrm{CML})$ slabs $(a<0)$ to double-positive, conventional 504 PML slabs $(a>0)$. This feature is explained by the resonant 505 nature of the CML with $a<0$. That is why we are focusing on 506 the case of CML $(a<0)$ rather than the conventional uniaxial 507 PML $(a>0)$. With the purple dots, we show (in the DNG 508 cases $a<0$ ) the points on each curve for which the normal 509 permittivity becomes lossless (it is lossy on the left side of 510 the dots and active on the right side). In other words, the dots 511 indicate the equality $\delta=\frac{b}{a^{2}+b^{2}} \Rightarrow a=-\sqrt{\frac{b}{\delta}-b^{2}}$, which is 512 valid (within the considered ranges of $a$ ) only for the three of 513 the four curves of Fig. 5(a) [and for none of Fig. 5(b)]. It is clear 514 that when one moves along that "ultimate passivity boundary" ${ }_{515}$ (where none of the permittivity/permeability components are 516 active) defined by the aforementioned successive purple dots, 517 takes a decreasing $R$ both for increasing $\delta$ and for increasing 518 $a<0$ (when $|a<0|$ becomes smaller). We can also conclude 519 that even when the medium is lossy for any direction of the 520 fields, the radiation enhancement is significant. 521

Most importantly, these results prove that the radiation 522 enhancement due to strong coupling of resonant surface modes 523 to the far-field modes is orders of magnitude higher than 524 possible reduction of radiation of propagating modes into the 525 far zone. Recall that in the absence of the scatterers and $\delta \rightarrow 0, \quad 526$ the CML slab is perfectly matched to free space. For small ${ }_{527}$ values of $b$, all propagating modes are fully reflected, and we 528 clearly see that adding pins makes the reflected fields more ${ }_{529}$ than two orders of magnitude stronger than reflected from a 530 conventional perfect reflector. Furthermore, $R$ is larger when $\delta 531$ is closer to zero which is anticipated by the limiting expression 532 of (3) in the DNG case. The best results are recorded when $a \quad 533$ is negative but close to zero (much larger than -1, namely, for 534 $-1 \ll a<0$ ) where the radiation enhancement is giant and 535 practically independent from $\delta$. In Fig. 5(b), we represent $R{ }_{536}$ as a function of $a$ for several loss parameters $b$. Again, we ${ }_{537}$ note that the cluster works only in the CML case, where it 538 does a very good job $(R>50$ on average). Finally, a smaller 539 imaginary part $b$ (with fixed $\delta>0$ ) favors the increase in the 540 radiated power achieved with the cylindrical vessels. 

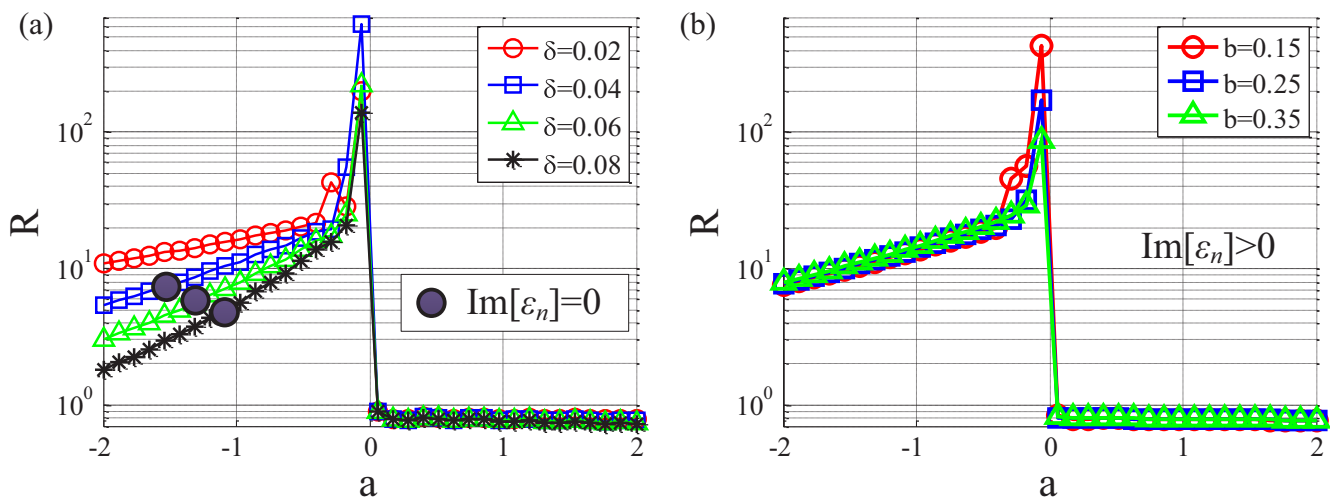

FIG. 5. The radiation enhancement ratio $R$ as a function of the real part of the transversal components $a=\operatorname{Re}\left[\varepsilon_{t}\right]=\operatorname{Re}\left[\mu_{t}\right]$ for (a) several perturbation parameters $\delta=\operatorname{Im}\left[1 / \varepsilon_{t}-\varepsilon_{n}\right]$ of the normal component of the material parameters (with $b=0.1$ ) and (b) several values of the imaginary part $b=-\operatorname{Im}\left[\varepsilon_{t}\right]=-\operatorname{Im}\left[\mu_{t}\right]$ (with $\delta=0.03$ ). Plot parameters: $r=\lambda_{0} / 200, N=80, g=\lambda_{0} / 20, L=3 \lambda_{0}, \theta=90^{\circ}$.

542 The singular behavior of the radiated power in the limit ${ }_{543} a \rightarrow 0^{-}$can be explained by considering the quality factor 544 of the resonating surface modes. To do that, we calculate the 545 equivalent inductance $L_{\mathrm{CML}}$ and resistance $R_{\mathrm{CML}}$ considering 546 the wave impedance $Z$ of the CML medium (2); similarly, 547 one can find expressions for the capacitive effect of free space ${ }_{548} C_{\mathrm{VAC}}$ by evaluating $Z_{0}$. If one assumes that $\delta>0$ (to ensure 549 overall passivity), evanescent modes $\left|k_{t}\right|>k_{0}$ (for which the 550 interesting phenomena happen) and $a<0$ (to have resonance), 551 the quality factor of the equivalent $R L C$ series circuit takes 552 the form

$$
\begin{aligned}
& Q=\frac{1}{R_{\mathrm{CML}}} \sqrt{\frac{L_{\mathrm{CML}}}{C_{\mathrm{VAC}}}} \Rightarrow \\
& Q \cong \frac{\sqrt{2\left(k_{t}^{2}-k_{0}^{2}\right)}}{k_{t}^{2}} \frac{\sqrt{2\left(k_{t}^{2}-k_{0}^{2}\right)+b \delta k_{t}^{2}}}{(-a) \delta}, \delta \rightarrow 0^{+} .
\end{aligned}
$$

553 It is easy to see that the loss parameter $R_{\mathrm{CML}}$ is proportional to $554(-a) \delta$ in this case. Thus, for a fixed level of losses in the CML 555 slab (measured by $\delta$ ), the quality factor behaves as $1 / a$ for $a \rightarrow$ ${ }_{556} 0^{-}$. Figure 6 shows the values of the quality factor on the plane

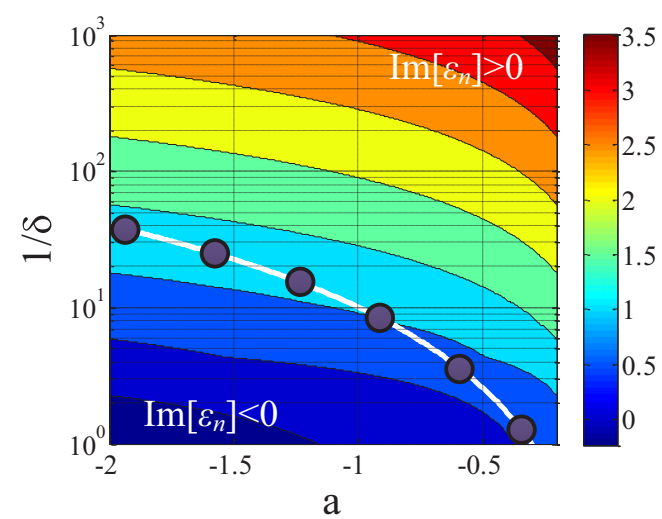

FIG. 6. The decimal logarithm of the quality factor of the equivalent circuit $\log Q$ with respect to the real part of the transversal components $a=\operatorname{Re}\left[\varepsilon_{t}\right]=\operatorname{Re}\left[\mu_{t}\right]$ and the inverse perturbation parameter $1 / \delta=1 / \operatorname{Im}\left[1 / \varepsilon_{t}-\varepsilon_{n}\right]$. Plot parameters: $k_{t}=1.5 k_{0}$, $b=0.1$. $(a, 1 / \delta)$ in the region $-2<a<0.2,1<1 / \delta<100$. It is clear 557 that $Q$ obtains huge magnitudes when $|a|, \delta$ are very small for 558 the CML scenario, namely, under the assumption of $a<0$. The 559 ultimate passivity boundary, along which we have a nonactive 560 $\varepsilon_{n}\left(\operatorname{Im}\left[\varepsilon_{n}\right]=0\right)$, is indicated by a white line with purple dots. It 561 divides the map $(a, 1 / \delta)$ into two regions: one upper right which 562 corresponds to active normal permittivity $\left(\operatorname{Im}\left[\varepsilon_{n}\right]>0\right)$ and 563 one lower left which concerns a passive normal permittivity 564 $\left(\operatorname{Im}\left[\varepsilon_{n}\right]<0\right)$.

In Fig. 7(a), we depict the variations of the radiation 566 enhancement $R$ with respect to the perturbation parameter 567 $\delta=\operatorname{Im}\left[\frac{1}{\varepsilon_{t}}-\varepsilon_{n}\right]=\operatorname{Im}\left[\frac{1}{\mu_{t}}-\varepsilon_{n}\right]$ for several values of the real 568 parts $a$ of the transversal constituent components. When $\delta>0,569$ namely, when the structure is overall passive, the effect of the 570 radiation vessels becomes weaker and weaker for increasing $\delta, 571$ which is also obvious from Fig. 5(a). Note, however, that when 572 the real part of the transversal permittivity approaches zero 573 from negative values, radiated power enhancement remains 574 substantial even for rather large positive $\delta$, that is, for rather 575 high overall losses in the system. On the other hand, when 576 $\delta<0$, the structure is overall active and the whole slab acts 577 as an additional power source; that is why the variations 578 are sharper and more parameter dependent. In particular, $R \quad 579$ possesses substantially oscillating and, on the average, much 580 higher values when $\delta<0$; in addition, the fluctuations are 581 weaker and the output more stable when $a$ is negative but 582 close to zero. It should be pointed out that these "spikes" ${ }_{583}$ in the response of the active structures reminds of electrical 584 thickness resonances combined with a proper excitation. As 585 it will be illustrated later in the analysis, such behavior is not 586 attributed mainly to the presence of a random grid of pins but ${ }_{587}$ to the fact that the grounded slab is active and infinite in size. 588 Again, one can observe the behavior of the system along the 589 "ultimate passivity limit" (purple dots) which indicate once 590 again that the functions $R=R(\delta>0)$ and $R=R(a<0)$ are 591 decreasing.

In Fig. 7(b), the change of $R=R(\delta)$ is shown for various 593 $b=\operatorname{Im}\left[\varepsilon_{t}\right]=\operatorname{Im}\left[\mu_{t}\right]$. The radiation enhancement is almost 594 independent from the imaginary part $b$ in the passive scenario, 595 while, similarly to Fig. 7(a), shaky response is observed when 596 $\delta<0$. It appears that when the system is overall active, one ${ }_{597}$ can find specific narrow intervals of $\delta$ where extremely high 598 

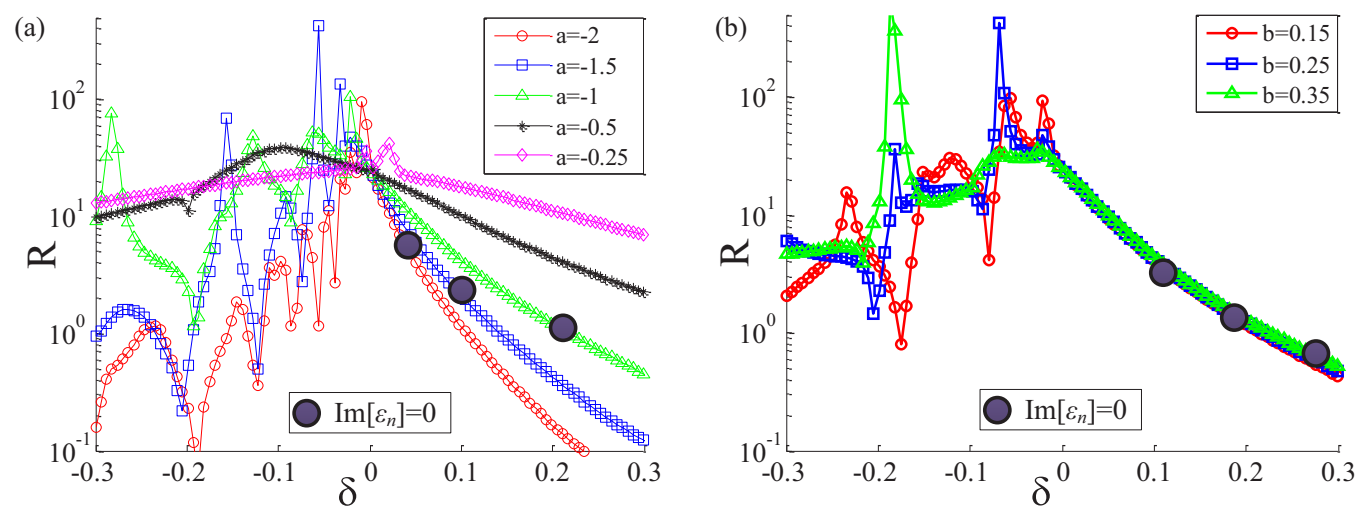

FIG. 7. The radiation enhancement ratio $R$ as function of the perturbation parameter $\delta=\operatorname{Im}\left[1 / \varepsilon_{t}-\varepsilon_{n}\right]$ for (a) various real part of transversal components $a=\operatorname{Re}\left[\varepsilon_{t}\right]=\operatorname{Re}\left[\mu_{t}\right]$ (with $b=0.2$ ) and (b) various imaginary parts $b=-\operatorname{Im}\left[\varepsilon_{t}\right]=-\operatorname{Im}\left[\mu_{t}\right]$ (with $a=-1$ ). Plot parameters: $r=\lambda_{0} / 200, N=80, g=\lambda_{0} / 20, L=3 \lambda_{0}, \theta=90^{\circ}$.

599 radiation is achieved regardless of the inherent losses $b$ along 600 the transversal directions.

601 As far as the rapid oscillations of the device response for $602 \delta<0$ are concerned, we show in Fig. 8(a) the reflection 603 coefficient when the radiation vessels are absent and the 604 structure is illuminated by an obliquely incident propagating 605 plane wave. In Fig. 8(a), we represent the decimal logarithm 606 of the reflection coefficient with respect to the perturbation 607 parameter of the imaginary part $\delta$ and the incidence angle. 608 Apparently, the reflection coefficient can be larger than unity 609 for the active case $(\delta<0)$; furthermore, sharp maxima are 610 recorded for the same half-plane close to the grazing angle. 611 In other words, similar "spikes" as those appearing in Fig. 7 612 constitute a characteristic of the active structure even though 613 no evanescent modes are considered (real incidence angles) 614 and no radiation vessels are used. They are just resonances 615 of an infinite active slab which pumps energy to the system 616 occurring at specific excitation directions. To better understand 617 how these oscillations depend on the real part of the transversal 618 permittivity/permeability of the CML, we show [Fig. 8(b)] the 619 average magnitude of the aforementioned reflection coefficient 620 (with respect to the real incidence angle) as a function of $\delta$ for ${ }_{621}$ several $a$. It is clear that the number of peaks increases for more negative $a$ and most of them are exhibited for $\delta \rightarrow 0^{-} ;{ }_{622}$ such a conclusion is compatible with the variations in Fig. 7(a). ${ }_{623}$ Finally, the curve spikes in Fig. 8(b) do not appear at the same $\delta \quad 624$ as those in Fig. 7(a) since they correspond to different systems 625 (excitation and structure); however, the inherent tendency of 626 the active slab $(\delta<0)$ towards abruptly changing response is 627 demonstrated in both cases.

A more systematic approach which proves the necessity 629 of an overall active slab $(\delta<0)$ in order to have sharp 630 maxima comes from the corresponding transmission-line 631 model of the grounded CML slab. The reflection coefficient 632 of a vessel-free structure [which is represented in Fig. 8(a)] 633 is written in terms of the TM wave impedances (2) as 634 follows: $R C=\frac{Z_{0}-Z \tanh \left[\kappa\left(k_{t}\right) L\right]}{Z_{0}+Z \tanh \left[\kappa\left(k_{t}\right) L\right]}$. The quantity $R C$ has large (or 635 infinite) magnitude, which imply sharp variations, when its 636 denominator takes small (or zero) values. It is more feasible 637 when the argument of hyperbolic tangent is an imaginary 638 number; it is then replaced by a trigonometric tangent whose 639 range is infinite and thus the equality $Z_{0}+Z \tanh \left[\kappa\left(k_{t}\right) L\right]=0 \quad 640$ becomes easier to get (even approximately) satisfied. Such 641 a condition can only be fulfilled when quantity under the 642 square root of $\kappa\left(k_{t}\right)$ is negative and thus purely real, namely, 643 when $\operatorname{Im}\left[\kappa^{2}\left(k_{t}\right)\right]=0 \Rightarrow k_{t}^{2}=2 b k_{0}^{2}\left(\frac{1}{2 b-\left(a^{2}+b^{2}\right) \delta}-\delta\right)$. For this 644
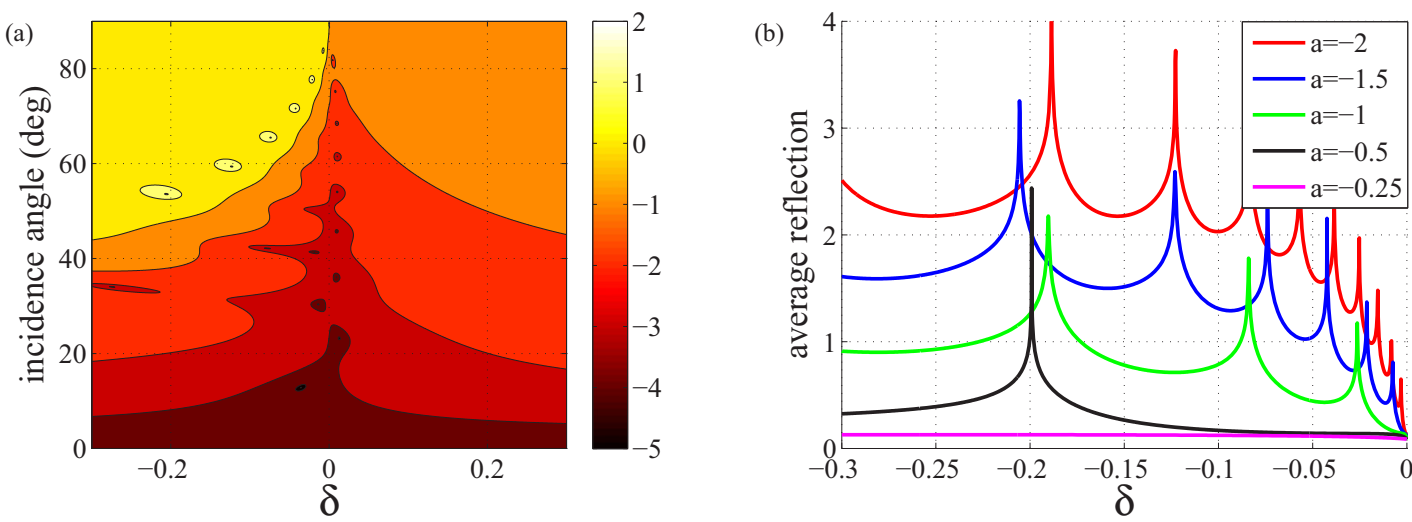

FIG. 8. (a) The decimal logarithm of the magnitude of the reflection coefficient of the vessel-free structure as function of the perturbation of the imaginary part $\delta$ and the incidence angle (with $a=-1.5$ ). (b) The average magnitude of the reflection coefficient over all the real incidence angles as function of the perturbation $\delta$ for various real parts of transversal components $a$. Plot parameters: $b=0.2, L=3 \lambda_{0}$. 

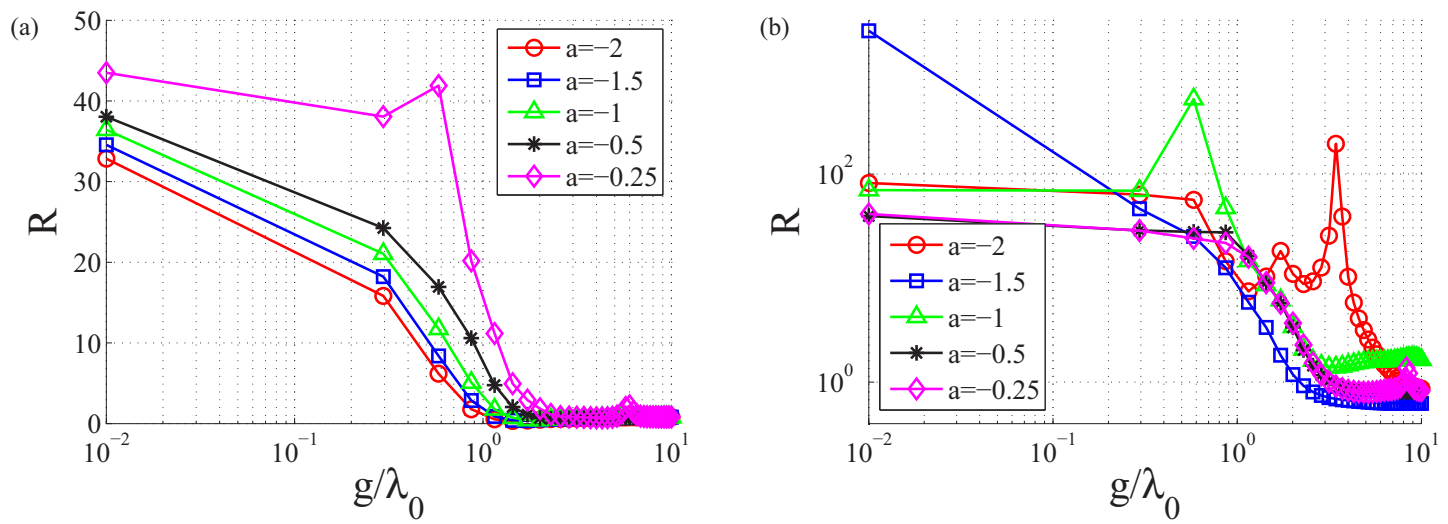

FIG. 9. The radiation enhancement ratio $R$ for various values of the real part of transversal components $a=\operatorname{Re}\left[\varepsilon_{t}\right]=\operatorname{Re}\left[\mu_{t}\right]$ as a function of the electrical distance of the source from the interface $g / \lambda_{0}\left(L=3 \lambda_{0}\right)$. (a) Overall passive CML with $\delta=0.02$. (b) Overall active CML with $\delta=-0.02$. Plot parameters: $b=0.2, r=\lambda_{0} / 200, N=80, \theta=90^{\circ}$.

645 direction of excitation, the propagation constant in the CML 646 slab is real and takes the form $\kappa \cong k_{0} \frac{a^{2}+b^{2}}{\sqrt{2 b}} \sqrt{\delta}$ for small $\delta \rightarrow 0$. 647 It is now clear that the only way for the quantity $\tanh \left[\kappa\left(k_{t}\right) L\right]$ 648 to have the sharp variations of the trigonometric tangent is to 649 use an overall active structure with $\delta<0$ (given that $b>0$ ).

650 In Fig. 9, we identify the influence of the location of the 651 primary source in representative passive and active scenarios 652 ( $\delta= \pm 0.02$ ). In Fig. 9(a), we can see that the radiation falls 653 rapidly as the primary source gets distant from the air-CML 654 slab interface because the evanescent part of the exciting 655 field gets weaker. However, especially in the active case 656 shown in Fig. 9(b), radiation enhancement remains significant 657 even when the distance to the source is much larger than 658 the wavelength. As indicated above, in the active case the 659 enhancement factor $R$ takes, on the average, higher values 660 and exhibits a less monotonic behavior as a function of the 661 geometrical and material parameters of the configurations.

\section{B. Radiation patterns}

Apart from the macroscopic insight offered by the radiation664 enhancement metric $R$, one can understand many features by 665 the introduced radiation-enhancing mirrors. The represented 666 quantities are normalized by $\left|h_{\text {inc }}(0)\right|=k_{0} \omega q / 4$. The incident 667 field in the far region $h_{\text {inc }}(\varphi)$ is evaluated by (7), the back- 668 ground field $h_{\text {inc }}(\varphi)+h_{\text {sec }}(\varphi)$ is given by $(8)$, and the total field 669 in the presence of the cylinders $h_{\text {inc }}(\varphi)+h_{\text {sec }}(\varphi)+h_{\text {scat }}(\varphi)$ is 670 computed using (18).

In Fig. 10, we illustrate two characteristic cases: one passive 672 [Fig. 10(a)] and one active [Fig. 10(b)]. The far-field patterns 673 are represented in the case that the grounded CML slab is 674 nearly fully reflecting propagating waves (we select the dissi- 675 pation parameter $b=0$ and the CML loss factor $\delta$ is small). 676 The three curves compare the far-field pattern of the primary 677 source into free space (green), the pattern for the CML slab 678 without perturbing pins (blue), and the CML slab with radia- 679 tion enhancing vessels (red). The green curve is simply the pat- 680 tern of a dipole line source, with the maximum in the broadside 681 direction [in both Figs. 10(a) and 10(b)]. CML slab without 682 pins basically acts as a reflector for the propagating part of the 683 incident spectrum and its response gets substantially enhanced 684 along the grazing-angle directions $\left(\varphi \cong 90^{\circ}, 270^{\circ}\right)$ for the 685 active scenario. We can see that for the passive mirror the max- 686 imum enhancement of the field strength equals 2 (fourfold in 687 terms of power), which takes place for directions along which 688
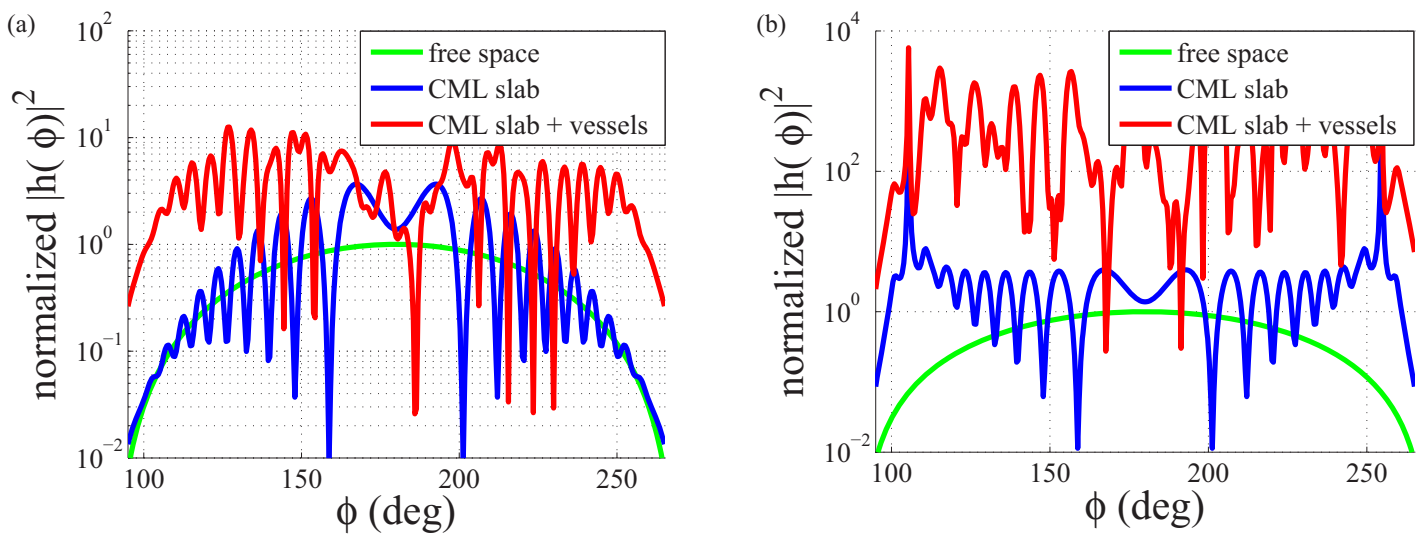

FIG. 10. The azimuthal profiles $|h(\varphi)|^{2}$ (normalized by $\left|h_{\text {inc }}(0)\right|^{2}=k_{0}^{2} \omega^{2} q^{2} / 16$ ) of the incident field, the background field and the total field as functions of angle $\varphi$ for: (a) a passive scenario $(\delta=+0.01)$ and (b) an active scenario $(\delta=-0.01)$. Plot parameters: $a=-2, b=0$, $L=3 \lambda_{0}, g \lambda_{0} / 10, r=\lambda_{0} / 100, N=80, \theta=90^{\circ}$. 

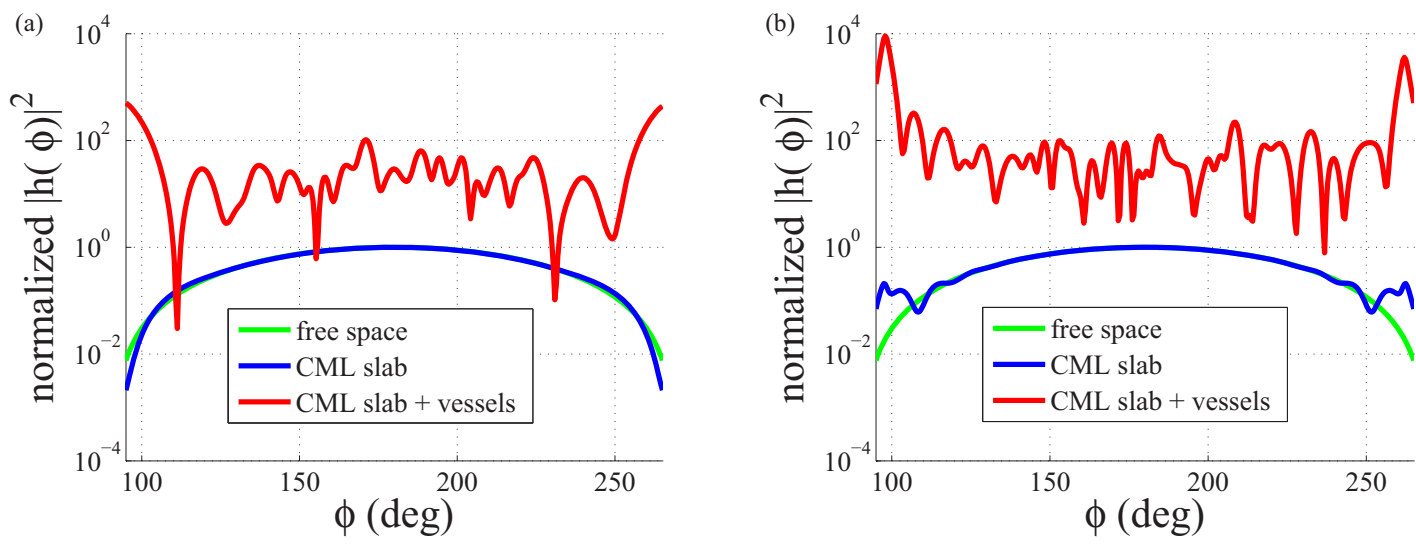

FIG. 11. The azimuthal profiles $|h(\varphi)|^{2}$ [normalized by $\left|h_{\text {inc }}(0)\right|^{2}=k_{0}^{2} \omega^{2} q^{2} / 16$ ] of the incident field, the background field, and the total field for (a) $a=-0.25, \delta=0.02, L=2.557 \lambda_{0}, g=\lambda_{0} / 20(R \cong 84)$ and (b) $a=-1, \delta=-0.02, L=3 \lambda_{0}, g=0.0665 \lambda_{0}(R \cong 525)$. Plot parameters: $b=0.2, r=\lambda_{0} / 200, N=80, \theta=90^{\circ}$.

689 the reflected field sums up in phase with the field of the primary 690 source. This value is the maximal possible value of the reflected 691 field from any ideally reflecting planar mirror (with arbitrary 692 reflection phase). We clearly see that the radiation vessels 693 provide an additional radiation channel via the evanescent part 694 of the spectrum, and the radiated power is strongly enhanced, 695 well above the fundamental limit for any lossless mirror.

696 In Fig. 11, we represent the results also for a passive 697 [Fig. 11(a)] and an active [Fig. 11(b)] case which correspond 698 to high radiation enhancement $R$. Losses in the CML are 699 present $(b=0.2)$, so that the reflections of the propagating 700 part of incident waves are weak. That is why we see that the 701 blue and green curves nearly coincide for the passive CML. 702 The chosen value of $\delta=0.02$ leads to an enhancement in 703 radiation by a factor of $R \cong 84$. In this case, it is apparent that 704 the far-field response of the CML slab without the radiation 705 enhancing cluster is almost identical to the incident field, 706 which is anticipated from (8). However, when one puts the 707 randomly distributed vessels in the near field, the output power 708 of the antenna gets significantly amplified (and the pattern 709 becomes asymmetric with respect to $\varphi=180^{\circ}$ ). In Fig. 11(b), 710 corresponding to an active CML, we use $\delta=-0.02$ and the 711 enhancement is huge in all directions (the overall radiated 712 power enhancement factor $R \cong 525$ ).

${ }_{713}$ All the proposed structures used in the aforementioned 714 cases incorporate as the main component the CML medium, 715 a material whose effective properties are described above. For 716 this reason, the issue of actually realizing DNG structures 717 with controllable values of the longitudinal parameters may be 718 raised. To emulate the negative transversal permittivities and 719 permeabilities, one of the most promising configurations is the 720 so-called fishnet structure [27,28]; a multilayered structure of 721 perforated thin metal sheets. As far as the longitudinal direction 722 is concerned, one can insert into the formed holes rods of 723 negative permittivity materials or wire media to achieve the 724 desired response. A more detailed description on how materials 725 with CML properties may be constructed is contained in [13].

\section{CONCLUSIONS}

727 728 faces can fully reflect electromagnetic waves in the limit of negligible losses. In this case, the amplitude of waves radiated 729 by a source near the mirror can be doubled as compared with 730 the incident waves. This surface is thought to be "ideally 731 shiny." On the other hand, the reflection coefficient from 732 planar surfaces can be, in principle, made zero for all incident 733 propagating waves (any polarization and any incident angle). 734 In this case, all power of incident propagating plane waves 735 is absorbed and the surface is "ideally black," absorbing 736 maximum power and, reciprocally, emitting maximal heat 737 power according to the Plank law. In both these scenarios, 738 evanescent waves do not participate in power exchange 739 between far-zone external sources and the material body. $\quad 740$

In this paper, we have shown that perturbing the boundary 741 of an infinite planar surface which maintains resonant surface 742 modes, can in principle realize a planar reflector which reflects 743 more power than any ideally reflecting planar surface. Due 744 to perturbations, surface modes couple to propagating plane 745 waves and create additional channels for power exchange via 746 evanescent fields. Such perturbed resonant surface extracts 747 extra power from near sources and sends that into space. The 748 amplitude of the reflected field can be orders of magnitude 749 larger than the maximal value of 2 for any usual lossless 750 mirror. Making the perturbation lossy, it can become possible 751 to overcome the black-body limit even for planar surfaces. In 752 this scenario, the black body absorbs nearly all propagating 753 waves, while the perturbations provide coupling between the 754 resonant surface modes and an additional energy sink via 755 evanescent modes.

The perturbations are random at the wavelength scale 757 and nonresonant. In this configuration, the surface-averaged 758 currents induced in the perturbation objects (which could 759 partially reflect propagating waves and compromise their 760 absorption in the CML body) are small because they couple to 761 nonresonant propagating modes of the absorbing or reflecting 762 body. On the other hand, current components which vary fast 763 on the wavelength scale can be huge because they couple to 764 highly resonant CML body. These spatially inhomogeneous 765 resonant currents on the perturbations provide additional 766 channels for power exchange between the body and free-space 767 wave modes, allowing stronger reflections than from an ideal 768 reflector or more absorption than in the ideal black body. $\quad 769$ 
770 Analyzing the far-zone radiation patterns, we see that 771 there is an analogy between the revealed phenomena and 772 superdirectivity of antennas [29]. Superdirective radiators can 773 create a narrow beam with the directivity higher than that of the 774 same configuration which is uniformly excited [30]. However, 775 in the configuration which we have introduced here, it appears 776 that the planar reflector sends superdirective beams nearly 777 everywhere (pattern oscillations are determined by random 778 positions of radiation vessels). There is also a connection of 779 the revealed phenomena to the concept of perfect lens as a 780 slab of a lossless double-negative material [31]. The perfect 781 lens operation also exploits resonance of surface modes at an 782 interface between free space and a double-negative material. 783 In the perfect lens concept, high-amplitude reactive fields 784 at the entry interface are focused behind the lens thanks 785 to interactions between resonant modes of the two parallel 786 surfaces of the lens. In papers [12,13], it was shown how 787 the reactive energy of the resonant surface modes can be 788 fully absorbed. Here, we have shown that this energy can 789 be launched into space, creating super-reflectors and far-field 790 superemitters.

791 This study is relevant also to the technologies of wireless 792 power transfer. In order to ensure the fastest wireless energy 793 transfer from a source to the user, one needs to maximize the channel capacity for power transport. In communications 794 technologies, the concept of MIMO (multiple input, multiple 795 output) exploits the idea of sending signals via many different 796 rays which may reach the receiver. However, if we are 797 concerned with the task of energy transfer, still only one 798 mode is conventionally exploited, even if multiple antennas 799 are used to send power to the receiver. In the near-field 800 scenario, this is the magnetic dipole mode of receiving coil 801 antennas. In the far-field scenario, this is the propagating plane 802 wave (transverse electromagnetic, TEM) mode. Here, we have 803 shown how a multichannel wireless power transfer can be in 804 principle realized.

Although in this paper we have considered a particular 806 realization of surface perturbations in the form of a random 807 array of thin cylinders, the concept is general and the surface 808 can be perturbed in many various ways, for instance, simply 809 making the surface rough at the appropriate wavelength scale. 810 Likewise, the surface does not have to be planar or infinite: 811 properly perturbing the surface of a finite-size conjugately 812 matched body we can dramatically enhance its coupling 813 to electromagnetic fields in space. Discussed superemission 814 and superabsorption phenomena can potentially enable new 815 approaches to optimizing wireless transfer of energy or 816 information and in radiative heat transfer management.
[1] C. M. Watts, X. Liu, and W. J. Padilla, Metamaterial electromagnetic wave absorbers, Adv. Mater. 24, OP98 (2012).

[2] Y. Radi, C. R. Simovski, and S. A. Tretyakov, Thin perfect absorbers for electromagnetic waves: Theory, design, and realizations, Phys. Rev. Appl. 3, 037001 (2015).

[3] E. E. Narimanov and A. V. Kildishev, Optical black hole: Broadband omnidirectional light absorber, Appl. Phys. Lett. 95, 041106 (2009).

[4] Q. Cheng, T. J. Cui, W. X. Jiang, and B. G. Cai, An omnidirectional electromagnetic absorber made of metamaterials, New J. Phys. 12, 063006 (2010).

[5] S.-A. Biehs, M. Tschikin, and P. Ben-Abdallah, Hyperbolic Metamaterials as An Analog of a Blackbody in the Near Field, Phys. Rev. Lett. 109, 104301 (2012).

[6] C. Simovski, S. Maslovski, I. Nefedov, and S. Tretyakov, Optimization of radiative heat transfer in hyperbolic metamaterials for thermophotovoltaic applications, Opt. Express 21, 14988 (2013).

[7] N. Mohammadi Estakhri and A. Alù, Minimum-scattering superabsorbers, Phys. Rev. B 89, 121416(R) (2014).

[8] D.-H. Kwon and D. M. Pozar, Optimal characteristics of an arbitrary receive antenna, IEEE Trans. Antennas Propag. 57, 3720 (2009).

[9] C. F. Bohren and D. R. Huffman, Absorption and Scattering of Light by Small Particles (Wiley, Weinheim, 2007), p. 129.

[10] G. Kirchhoff, On the relation between the radiating and absorbing powers of different bodies for light and heat, Philos. Mag. 4, 1 (1860).

[11] M. Planck, The Theory of Heat Radiation, 2nd. ed. translated by M. Masius (P. Blakiston's Son, Philadelphia, 1914).

[12] S. I. Maslovski, C. R. Simovski, and S. A. Tretyakov, Overcoming black body radiation limit in free space:
Metamaterial superemitter, New J. Phys. 18, 013034 (2016).

[13] C. A. Valagiannopoulos, J. Vehmas, C. R. Simovski, S. A. Tretyakov, and S. Maslovski, Electromagnetic energy sink, Phys. Rev. B 92, 245402 (2015).

[14] Y. I. Bobrovnitskii, Impedance theory of sound absorption: The best absorber and the black body, Acoust. Phys. 52, 638 (2006).

[15] L. N. Zakhariev and A. A. Lemanski, Scattering of Waves by “Black” Bodies (Sovetskoje Radio 34, Moscow, 1972) (in Russian).

[16] S. D. Gedney, An anisotropic perfectly matched layer-absorbing medium for the truncation of FDTD lattices, IEEE Trans. Antennas Propag. 44, 1630 (1996).

[17] R. W. Ziolkowski, The design of Maxwellian absorbers for numerical boundary conditions and for practical applications using artificial engineered materials, IEEE Trans. Antennas Propag. 45, 656 (1997).

[18] F. L. Teixeira and W. C. Chew, General closed-form PML constitutive tensors to match arbitrary bianisotropic and dispersive linear media, IEEE Microwave Guided Wave Lett. 8, 223 (1998).

[19] S. A. Tretyakov and T. G. Kharina, The perfectly matched layer as a synthetic material with active inclusions, Electromagnetics 20, 155 (2000).

[20] N. Tedeschi, F. Frezza, and A. Sihvola, On the perfectly matched layer and the DB boundary condition, J. Opt. Soc. Am. A 30, 1941 (2013).

[21] V. G. Veselago, The electrodynamics of substances with simultaneously negative values of $\epsilon$ and $\mu$, Usp. Fiz. Nauk 92, 517 (1967) [Sov. Phys.-Usp. 10, 509 (1968)].

[22] C. A. Valagiannopoulos, M. S. Mirmoosa, I. S. Nefedov, S. A. Tretyakov and C. R. Simovski, Hyperbolic-metamaterial 
antennas for broadband enhancement of dipole emission to free space, J. Appl. Phys. 116, 163106 (2014).

[23] L. B. Felsen and N. Marcuvitz, Radiation and Scattering of Waves, IEEE Series on Electromagnetic Wave Theory (Wiley, Hoboken, NJ, 1972).

[24] C. A. Valagiannopoulos, On examining the influence of a thin dielectric strip posed across the diameter of a penetrable radiating cylinder, Prog. Electromagn. Res. C 3, 203 (2008).

[25] C. A. Valagiannopoulos, Study of an electrically anisotropic cylinder excited magnetically by a straight strip line, Prog. Electromagn. Res. C 73, 297 (2007).

[26] S. A. Tretyakov, Analytical Modeling in Applied Electromagnetics (Artech House, London, 2003).
[27] J. Valentine, S. Zhang, T. Zentgraf, E. Ulin-Avila, D. A. Genov, G. Bartal, and X. Zhang, Three-dimensional optical metamaterial with a negative refractive index, Nature (London) 455, 376 (2008).

[28] M. Kafesaki, I. Tsiapa, N. Katsarakis, Th. Koschny, C. M. Soukoulis, and E. N. Economou, Left-handed metamaterials: The fishnet structure and its variations, Phys. Rev. B 75, 235114 (2007).

[29] S. A. Schelkunoff, A mathematical theory of linear arrays, Bell Syst. Tech. J. 22, 80 (1943).

[30] R. C. Hansen, Fundamental limitations in antennas, Proc. IEEE 69, 170 (1981).

[31] J. B. Pendry, Negative Refraction Makes a Perfect Lens, Phys. Rev. Lett. 85, 3966 (2000). 